\title{
Slow Oscillations of Membrane Potential in Interneurons That Control Heartbeat in the Medicinal Leech
}

\author{
Edmund A. Arbas and Ronald L. Calabrese ${ }^{a}$ \\ Biological Laboratories, Harvard University, Cambridge, Massachusetts 02138
}

In the preceeding paper (Arbas and Calabrese, 1987), we identified several properties that contribute to the activity of neurons (HN cells) that control heartbeat in the medicinal leech. Premotor HN (7) interneurons, which do not generate the heartbeat rhythm, exhibit $\mathrm{Na}^{+}$-dependent fast action potentials, $\mathrm{Ca}^{2+}$-mediated plateau potentials in the absence of $\mathrm{Na}^{+}$, and hyperpolarization-activated "restorative" changes in membrane potential that depolarize the membrane potential on hyperpolarization due to injected currents or synaptic inhibition. HN interneurons of ganglia 3 and 4 (i.e., timing oscillator interneurons) exhibit all of the properties described for HN (7) interneurons and have the additional characteristic that they are connected in oscillatory circuits. Reciprocal oscillations in membrane potential occurred in the bilateral HN interneurons (3) and (4) in the presence of elevated $\mathrm{Ca}^{2+}$ that were independent of $\mathrm{Na}^{+}$-mediated action potentials. Their ability to oscillate in this way is based on 3 parameters: (1) production of a regenerative plateau potential by one of the pair of HN neurons in either ganglion, (2) inhibition of the contralateral HN neuron by the HN neuron in plateau, and (3) a phase transition mediated by escape from inhibition by the hyperpolarized HN neuron.

The conductances responsible for restorative membrane potential shifts activated by hyperpolarization during synaptic inhibition may mediate the escape from inhibition that times the phase transition of the $2 \mathrm{HN}$ neurons.

Defining mechanisms that promote rhythmicity in the activity of neurons and circuits is important to our understanding of the neural basis of rhythmic behavior in man and animals. Rhythmic pattern generating circuits in the CNS underlie virtually all rhythmic behavior (Delcomyn, 1980), and the interacting mechanisms that produce centrally generated rhythms include endogenous properties of particular neurons and the pattern and properties of their synaptic interactions (Selverston and Moulins, 1985; also see Roberts and Roberts, 1983, for reviews). Our studies are aimed at elucidating both the endogenous and synaptic properties of neurons that control heartbeat in the medicinal leech (Thompson and Stent, 1976a-c; Calabrese and Peterson, 1983; Arbas and Calabrese, 1987).

\footnotetext{
Received Dec. 11, 1986; revised May 11, 1987; accepted June 5, 1987.

Supported by NIH postdoctoral fellowship 5 F32 NS 06453 (to E.A.A.) and NIH Grant NS 24072 (to R.L.C.).

Correspondence should be addressed to Edmund A. Arbas, Arizona Research Laburatories, Division of Neurobiology, 611 Gould-Simpson Science Building, University of Arizona, Tucson, AZ 85721.

a Present address: Department of Biology, Emory University, 1555 Pierce Drive, Atlanta, GA 30322 .

Copyright (C) 1987 Society for Neuroscience $0270-6474 / 87 / 123953-08 \$ 02.00 / 0$
}

The source of the heartbeat rhythm lies in a subcircuit of the heartbeat pattern generator that resides in the 3rd and 4th segmental ganglia of the ventral nerve cord that has been termed the "timing oscillator" of the system (Peterson and Calabrese, 1982; Calabrese and Peterson, 1983). Interneurons of the timing oscillator (HN neurons) are interconnected by reciprocal inhibitory synapses (Fig. 1A), a circuit configuration that both coordinates firing into antiphasic bursts (Calabrese, 1979; Peterson, 1983a, b) (Fig. 1B) and promotes oscillation when combined with certain other endogenous properties (Brown, 1914; Perkel and Mulloney, 1974). Timing oscillator interneurons also show the capability for endogenous bursting when their major synaptic inputs are experimentally blocked (Calabrese, 1979).

We have demonstrated in the preceding paper (Arbas and Calabrese, 1987) that several intrinsic properties contribute to the activity of $\mathrm{HN}$ neurons of the heartbeat pattern generator, including (1) $\mathrm{Na}^{+}$-dependent fast action potentias; (2) a voltagedependent $\mathrm{Ca}^{2+}$ conductance that contributes to the formation of plateau potentials in the absence of $\mathrm{Na}^{+}$, and (3) hyperpolarization-activated restorative shifts in membrane potential that oppose the induced hyperpolarization. We examine here how these properties interact to promote oscillation of the timing oscillator network.

\section{Materials and Methods}

The preparations and methods used are identical to those described in Arbas and Calabrese (1987), with the exception that this report emphasizes recordings made from timing oscillator interneurons of ganglia 3 or 4.

\section{Results}

Properties of HN neurons of the timing oscillator

The conductances that mediate the plateau potentials formed in the absence of $\mathrm{Na}^{+}$and hyperpolarization-activated changes in membrane potential (Arbas and Calabrese, 1987) are properties of $\mathrm{HN}$ interneurons that may contribute significantly to their rhythmical activity. Another property of HN neurons that may contribute to the activity of the timing oscillator is their ability to release neurotransmitter in the absence of $\mathrm{Na}^{+}$-mediated action potentials (Thompson and Stent, 1976c; Nicholls and Wallace, 1978). Fvidence for all of these properties can be seen in recordings from interneurons of the timing oscillator bathed by normal saline (Fig. 1). Injection of small, constantcurrent hyperpolarizing pulses produces membrane potential changes with an initial maximum hyperpolarization followed by a time-dependent sag to more depolarized values (Fig. $1 B$ ). Release from hyperpolarization at the end of an injected pulse produces a response in which membrane potential jumps briefly to a plateau near $-30 \mathrm{mV}$ and then gradually returns to normal 
A.

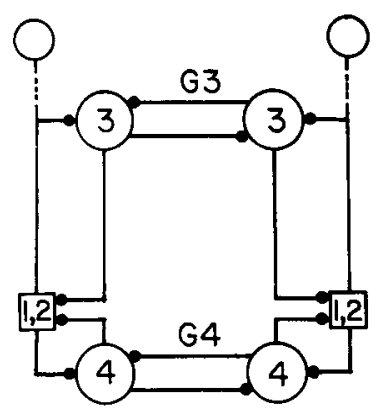

C1.

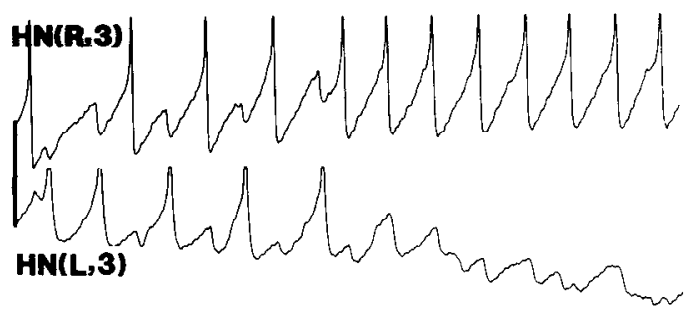

C2.

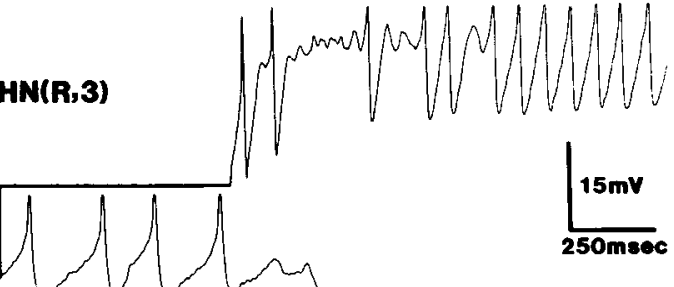

B. HN(L,3)

HN(R,3)
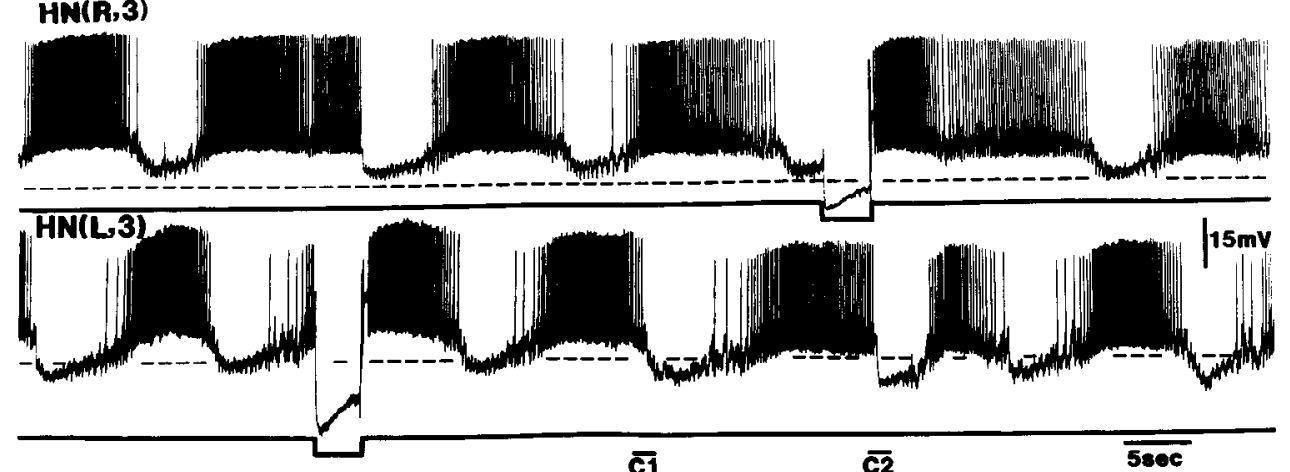

Figure 1. Connections of $\mathrm{HN}$ interneurons of the timing oscillator and their properties in normal saline. $A$, Schematic showing connectivity among HN interneurons in the timing oscillator. Open circles depict HN interneurons numbered by the ganglia in which they reside (G3 or G4); filled circles represent chemical inhibitory synaptic connections. Cells HN (1) and HN (2) are lumped together because their properties and connections are indistinguishable. Their primary input regions and spike initiation sites are in the fourth ganglion and are indicated separately from their somata by open squares. $B$. Activity of cells HN (3) in normal saline consists of bursts of action potentials that are locked into antiphasic coordination by the reciprocal inhibitory connections between them. When a constant-current hyperpolarizing pulse was injected into either cell, the membrane potential moved initially to a maximum value, then gradually relaxed to morc depolarized levels for the remainder of the pulse. On termination of injected pulses, both $\mathrm{HN}$ interneurons exhibited a plateau response in which the membane potential jumped briefly to a membrane potential near $-30 \mathrm{mV}$, which was followed by several seconds of accelerated firing. Dashed lines indicate a potential of $-50 \mathrm{mV}$; solid lines are current monitor traces. $C 1$ and $C 2$, Expanded records at higher gain of regions labeled correspondingly in $B$ that show a typical transition from a burst of activity in cell $\mathrm{HN}(\mathrm{L}, 3)$ to a burst of activity in cell $\mathrm{HN}(\mathrm{R}, 3)(\mathrm{Cl})$, and the transition induced by plateau formation on release from a hyperpolarizing pulse in $\mathrm{HN}(\mathrm{R}, 3)(C 2)$. Peaks of action potentials were clipped by the pen recorder.

baseline with accelerated firing of action potentials (Fig. $1 B$, C2). Similar plateaus in HN (7) interneurons appear to be mediated by an increased conductance to $\mathrm{Ca}^{2+}$ (Arbas and $\mathrm{Cal}-$ abrese, 1987). Formation of the plateau in either of the pair of $\mathrm{HN}$ interneurons of ganglia 3 or 4 is accompanied by an enhanced inhibition of the contralateral $\mathrm{HN}$ neuron (Fig. 1, Cl, C.2).

Elimination of fast $\mathrm{Na}^{+}$-dependent action potentials by substituting Tris for $\mathrm{Na}^{+}$in the bath and elevating $\mathrm{Ca}^{2+}$ concentration revealed the abilities of timing oscillator interneurons to form plateau potentials and release neurotransmitter in the absence of $\mathrm{Na}^{+}$-mediated action potentials more clearly (Fig. 2).

\section{Oscillation of HN interneurons}

Elevation of $\mathrm{Ca}^{2+}$ ion concentration and reduction of $\mathrm{Na}^{+}$in the bathing medium produces conditions in which timing os- cillator interneurons can oscillate for many minutes independently of the production of $\mathrm{Na}^{+}$-mediated action potentials and impulse-mediated synaptic transmission (Fig. 3). Oscillation of timing oscillator interneurons under these conditions is based on 3 parameters: (1) the production of a plateau potential by one HN neuron, (2) inhibition of the contralateral neuron by the release of transmitter during the plateau, and (3) a transition in state between the 2 neurons.

The transition between the plateau phase and the hyperpolarized phase can conceivably occur in at least 2 ways. The selftermination of a plateau could release the contralateral neuron from inhibition, permit it to rebound into a plateau of its own, and inhibit the previously depolarized neuron. Alternatively, the inhibited neuron might escape from inhibition, form a plateau, and terminate the plateau of the contralateral neuron through synaptic inhibition. Inspection of the recordings shown in Figure 3 suggests that the second mechanism was operating. 


\section{$25 \% \mathrm{Na} 5 \mathrm{mM} \mathrm{Ca}$}

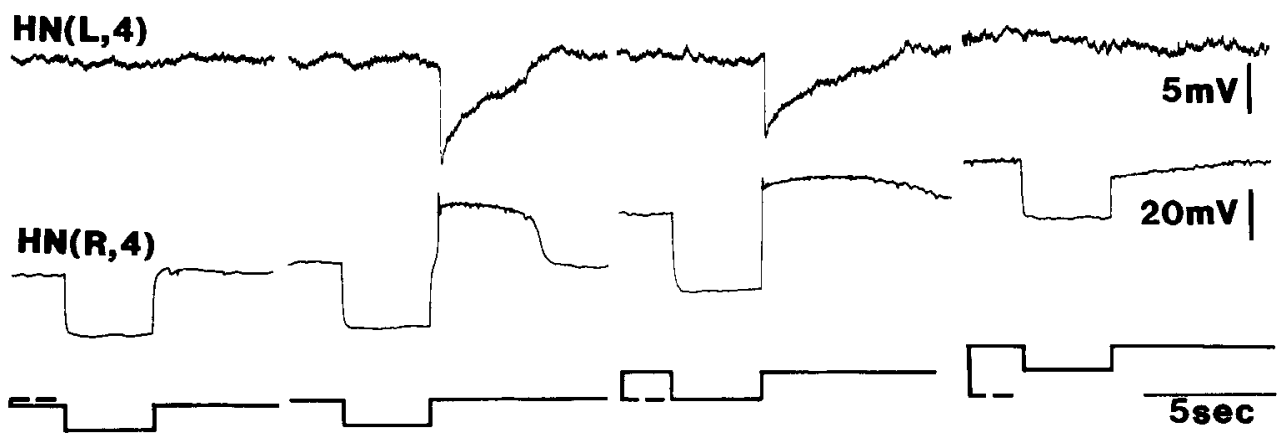

Figure 2. Transmitter release by $\mathrm{HN}$ interneurons in the absence of $\mathrm{Na}^{+}$-mediated action potentials. The ganglion was bathed by saline containing $25 \%$ of normal $\mathrm{Na}^{+}$and $5 \mathrm{~mm} /$ liter $\mathrm{Ca}^{2+}$. Cell $\mathrm{HN}(\mathrm{L}, 4)$ was held at a membrane potential level of $-56 \mathrm{mV}$ to prevent plateau formation, while constant-current hyperpolarizing pulses were injected into the $\mathrm{HN}(\mathrm{R}, 4)$ interneuron held at different potential levels by a bias current. Dashed lines associated with the current monitor traces indicate zero injected current. When neuron HN $(\mathbf{R}, 4)$ was maintained below threshold for plateau formation, release from the hyperpolarizing pulse caused no postsynaptic effect in the $H N(L, 4)$ neuron. When plateaus were formed in the HN $(R, 4)$ neuron, postsynaptic hyperpolarization of the $H N(L, 4)$ neuron was evident. If the $H N(R, 4)$ neuron was maintained at near - 30 mV, no plateau was formed and no postsynaptic response was elicited. Injected square pulses were $1.1 \mathrm{nA}$ in amplitude.

The membrane potential of the neuron in plateau phase was nearly constant during the peak of each plateau, whereas the membrane potential of the inhibited neuron gradually depolarized until a plateau was formed. To test the mechanism of the phase transition, a hyperpolarizing current pulse was injected into one of the oscillating pair of neurons to block a plateau just as it was about to be initiated (Fig. $3 B$ ). If plateau termination were the mechanism timing the phase transition, then blockade of the plateau in one neuron would not affect the timing of plateau termination in the contralateral neuron. In the experiment illustrated in Figure $3 B$, the plateau in the contralateral neuron ultimately did terminate, indicating that a mechanism for plateau repolarization does exist, but the repolarization was delayed. Thus, escape from inhibition and formation of the plateau by the inhibited neuron in each unperturbed cycle of oscillation seems to time phase transitions. Blocking plateau production in one $\mathrm{HN}$ neuron by injected current halted the activity of both neurons, indicating that the oscillation was based on the reciprocal inhibition between the 2 neurons and not some extrinsic influence. Removing the hyperpolarizing current initiated a plateau and restarted the oscillation.

The independence of plateau-based oscillation from $\mathrm{Na}^{+} \mathrm{ac}-$

A.

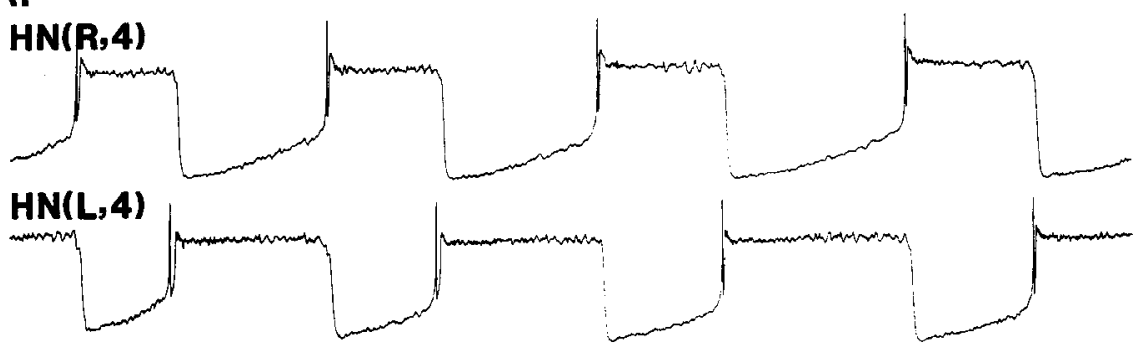

B.

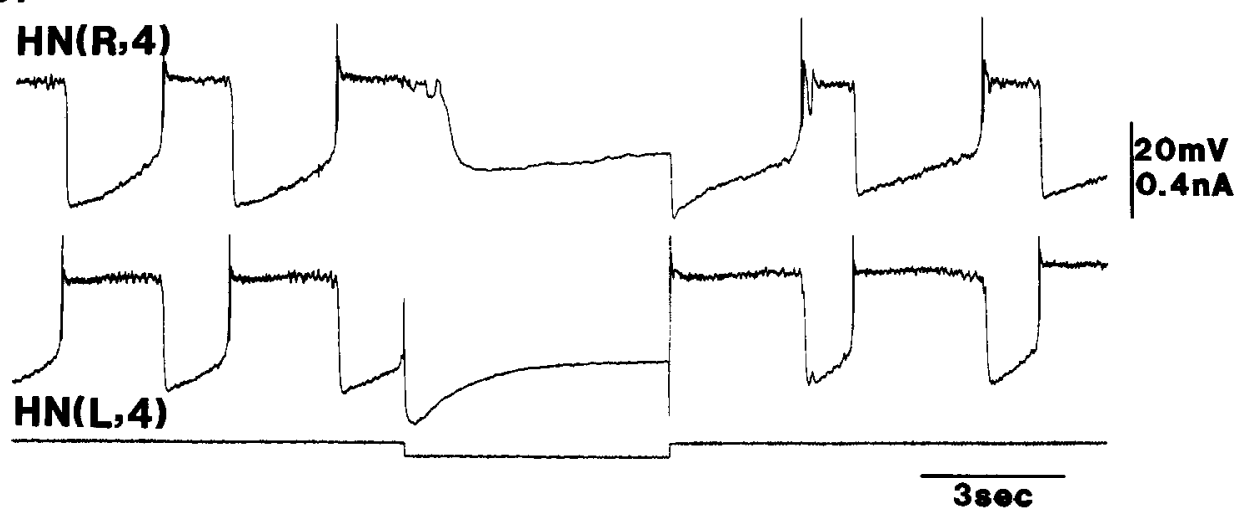

Figure 3. A, Sustained oscillation in a pair of timing oscillator interneurons independent of the production of $\mathrm{Na}^{+}$. dependent action potentials. $B$, Injection of a hyperpolarizing current pulse into neuron $\mathrm{HN}(\mathrm{L}, 4)$ that prevented formation of a plateau led to prolongation of the plateau in the contralateral HN cell. This indicates that escape from inhibition leading to plateau production timed the transition in states of the 2 cells. The preparation was bathed in $25 \% \mathrm{Na}^{+}, 20 \mathrm{~mm} /$ liter $\mathrm{Ca}^{2+}$. 

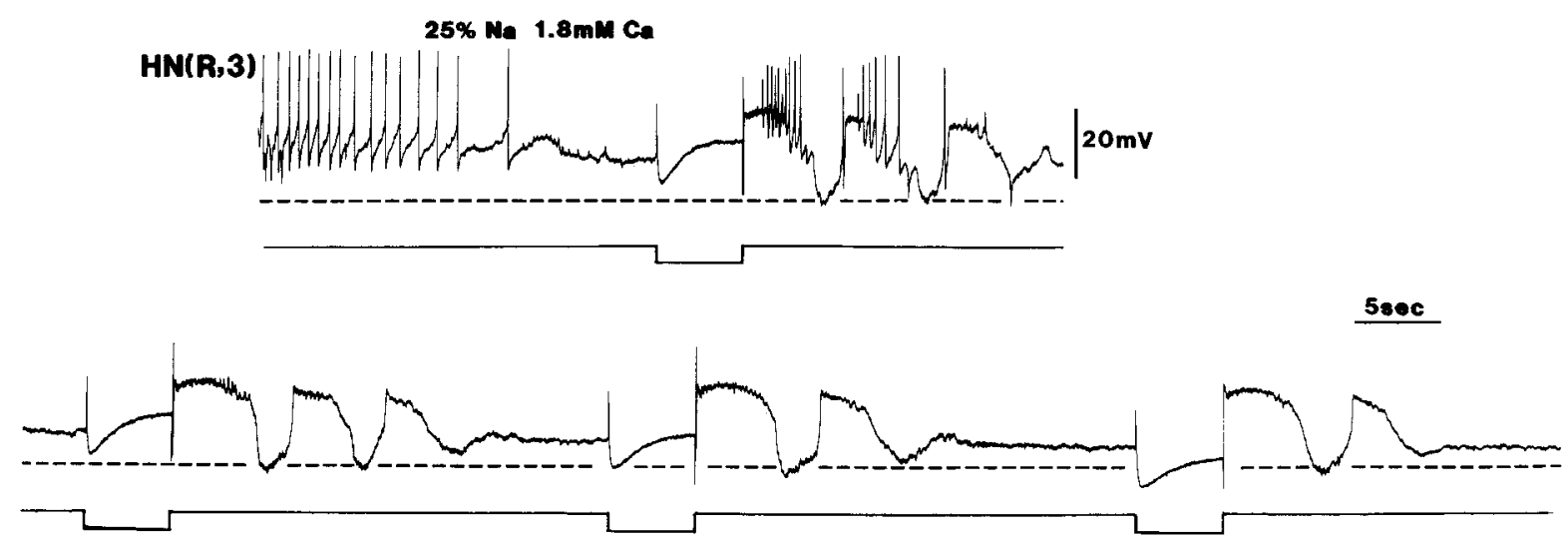

Figure 4. Oscillation in low- $\mathrm{Na}^{+}$saline in the presence of normal $\mathrm{Ca}^{2+}$. Recording from an $\mathrm{HN}(\mathrm{R}, 3)$ while washing in saline containing $25 \%$ $\mathrm{Na}^{+}$and normal $\mathrm{Ca}^{2+}$ showed that spontaneous oscillation between the pair of HN interneurons ceased in the low-Na+ saline (beginning of record). When a plateau and action potentials were activated following a hyperpolarizing pulse, the $H N(R, 3)$ underwent several cycles of oscillation in alternation with its contralateral homolog (indicated by the inhibition in the trace). Even when $\mathrm{Na}^{+}$action potentials ceased to be formed (in the lower traces of the continuous record), the HN cells underwent several cycles of oscillation following termination of an injected pulse. Injected pulses were $0.1 \mathrm{nA}$.

tion potentials is clear in recordings such as shown in Figure 3, where fast $\mathrm{Na}^{+}$action potentials were eliminated by substituting Tris for most of the $\mathrm{Na}^{+}$in the bath and $\mathrm{Ca}^{2+}$ concentration was elevated to $20 \mathrm{~mm} /$ liter. Under these conditions, slower $\mathrm{Ca}^{2+}$-dependent spikes similar to those elicited in $\mathrm{HN}(7)$ interneurons in elevated $\mathrm{Ca}^{2+}$ salines (Arbas and Calabrese, 1987) often preceded the plateaus. Oscillations of $\mathrm{HN}$ interneuron pairs whose activity included both the $\mathrm{Ca}^{2+}$-dependent plateaus and $\mathrm{Na}^{+}$-mediated action potentials were obtained when $\mathrm{Ca}^{2+}$ was elevated in salines containing normal $\mathrm{Na}^{+}$or in salines in which $\mathrm{Na}^{+}$was reduced, but only to levels insufficient for blockade of spiking (Figs. 4, 7). Lowering $\mathrm{Na}^{+}$in the bath typically reduced the frequency of action potentials as well as their amplitude and allowed plateaus to form. Elevating the concentration of divalent ions in the bath also had the effect of raising the threshold for $\mathrm{Na}^{+}$action potentials (Frankenhaeuser and Hodgkin, 1957). Plateau-based oscillations often began spontaneously and lasted for many minutes in the presence of elevated $\mathrm{Ca}^{2+}$. Oscillations of $\mathrm{HN}$ cells were observed when $\mathrm{Na}^{+}$ ion concentration in the saline was reduced without elevating $\mathrm{Ca}^{2+}$ from normal (Figs. 4, 7), but such oscillations typically lasted for shorter lengths of time than those in the presence of elevated $\mathrm{Ca}^{2+}$.

Where both action potentials and plateaus contributed to the oscillation of $\mathrm{HN}$ cells, production of action potentials often seemed to trigger the formation of plateaus, and afterhyperpolarizations of action potentials often terminated plateaus before the end of each activity cycle (Figs. 5, 7). Where this occurred, the transition of an $\mathrm{HN}$ interneuron from its depolarized active state (plateau and spikes) to its silent phase was typically mediated by the recovery from inhibition of the contralateral $\mathrm{HN}$ neuron and its activation of spiking or plateau (Figs. 5, 7).

Plateau-based oscillation could be halted by the injection of sustained hyperpolarizing current into one of an oscillating pair of HN neurons (Figs. $3 ; 5, A, C$ ). If the injected current was reduced to zero without forming a plateau potential, the membrane potential could be restored to a level suprathreshold for action potentials without reinitiating the oscillation (Fig. $5 B$ ). Under these conditions, there was no evidence of spontaneous production of plateau potentials, although spiking occurred. If the hyperpolarizing current was removed rapidly, a plateau would form and the oscillation would be triggered (Figs. $4 ; 5 \mathrm{C}$ ).

\section{Escape from inhibition}

Transitions from the depolarized active state to the hyperpolarized silcnt statc in strongly oscillating pairs of $\mathrm{HN}$ neurons are timed by escape from inhibition of the hyperpolarized member of the pair. Where the oscillation is based on $\mathrm{Ca}^{2+}$-dependent plateaus in the absence of $\mathrm{Na}^{+}$action potentials, recovery from inhibition is followed by the formation of a plateau. The escape of one $\mathrm{HN}$ neuron from plateau-mediated inhibition during oscillation can be mimicked when one of a nonoscillating pair of HN neurons is depolarized by injected current and synaptically inhibits its contralateral homolog (Fig. 6).

How might the escape from inhibition be mediated? One mechanism that may operate is the waning of synaptic transmission due to factors such as depletion of readily releasable transmitter stores during the plateau, desensitization of postsynaptic receptors, or some combination of these. Another possible mechanism is the initiation of restorative shifts in membrane potential (Arbas and Calabrese, 1987) by the hyperpolarization due to synaptic inhibition. If the latter mechanism is capable of activating the plateau or burst that follows each hyperpolarized silent period, then it should be possible to initiate oscillation in nonoscillating pairs of $\mathrm{HN}$ cells by activating the restorative changes in membrane potential with injected hyperpolarizing current. Figure 7 shows the result of such a manipulation. Injection of a hyperpolarizing pulse sufficient to activate the restorative sag in membrane potential in one $\mathrm{HN}$ neuron of a noncycling pair produced "breakaway" oscillation of the network when the restorative sag led to the formation of a plateau (Fig. 7).

Thus, hyperpolarization due to synaptic inhibition appears to have multiple effects on the postsynaptic cell. It not only repolarizes the active $\mathrm{HN}$ neuron and thereby halts its release of transmitter but also activates a time-dependent trigger for the next plateau by activating conductances responsible for the restorative sag in membrane potential. 
A. $\mathrm{HN}(\mathrm{R}, 4)$

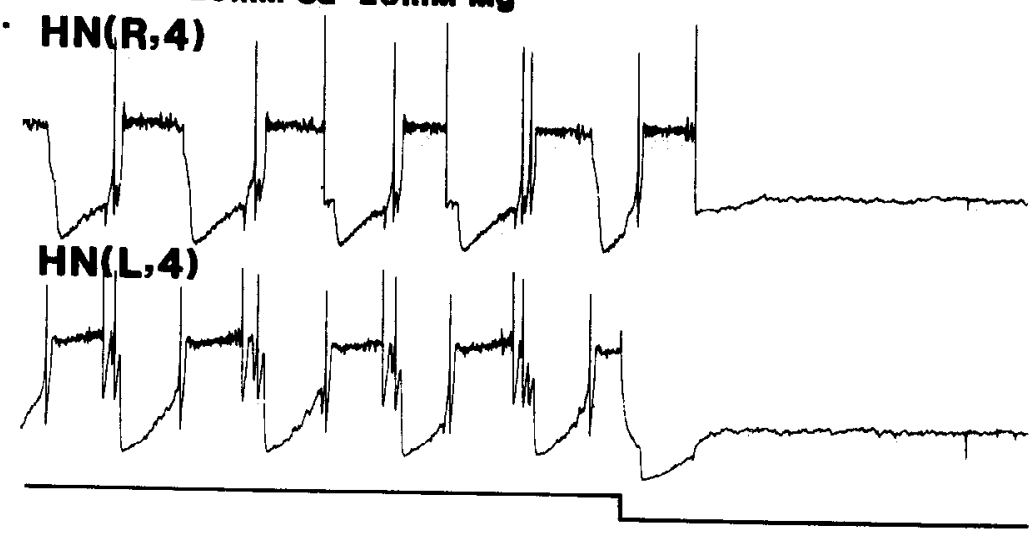

B.
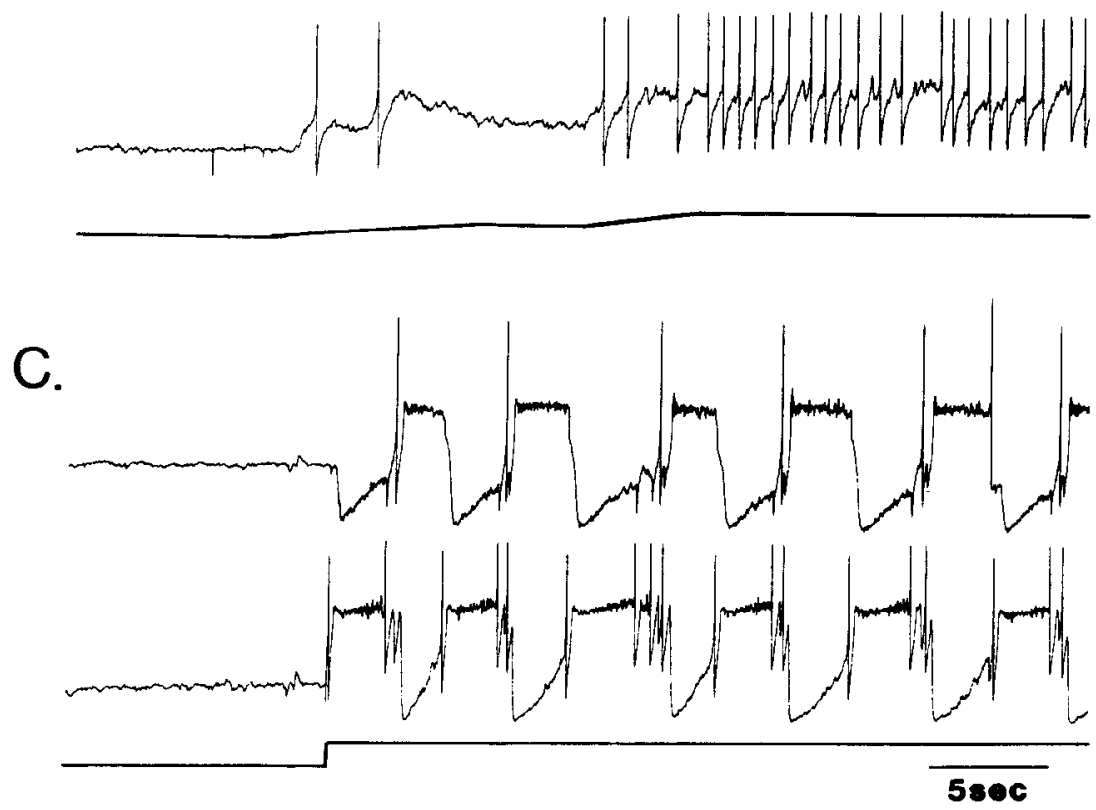

Figure 5. A, Oscillation in a pair of timing oscillator interneurons in the presence of $20 \mathrm{~mm} /$ liter each of $\mathrm{Ca}^{2+}$ and $\mathrm{Mg}^{2+}$ and normal $\mathrm{Na}^{+}$can be terminated by injection of a sustained hyperpolarizing current into one $\mathrm{HN}$ cell. $B$. When the hyperpolarization was released without forming a plateau, the HN cell was restored to a potential level where action potentials were produced but without endogenous production of plateaus. $C$, If hyperpolarization similar to that in $A$ was released suddenly, a plateau potential was initiated that set off the plateau-based oscillation between the two neurons.

\section{Restorative shifts in membrane potential are not necessary for all types of oscillation}

The conductances responsible for the restorative sag in membrane potential induced by hyperpolarization of $\mathrm{HN}$ interneurons are sensitive to blockade by low concentrations of $\mathrm{Cs}^{+}$ions (Arbas and Calabrese, 1987), thereby providing a means to test their role in the normal oscillation of $\mathrm{HN}$ cell pairs. Washing saline containing $5 \mathrm{~mm} /$ liter $\mathrm{Cs}^{+}$over an isolated ganglion (3) slowed the rate of cycling of the HN (3) neurons but did not eliminate oscillation completely (Fig. 8). Thus, although the restorative sag in membrane potential is likely to play an important role in the timing of the oscillation, other parameters appear to be capable of shaping the activity of $\mathrm{HN}$ neurons into alternating bursts in its absence.

\section{Discussion}

The circuits formed by $\mathrm{HN}$ (3) and HN (4) neuron pairs each constitute "half-center" oscillators, the simplest oscillatory net- work that can be made of neurons lacking individual endogenous activity (Brown, 1914). Although $\mathrm{HN}$ neurons have the ability to oscillate independently when their synaptic linkages are experimentally uncoupled (Calabrese, 1979), these rhythmical properties are typically not expressed under the conditions of the present study. Thus, the plateau-based oscillation that HN neurons express in isolated ganglia derives solely from the half-center configuration. The oscillation depends on several important endogenous properties, as well as the reciprocally inhibitory circuit configuration. Plateau potential formation mediates the release of inhibitory neurotransmitter in the absence of $\mathrm{Na}^{+}$spikes. Similar properties are known to be embedded in other oscillatory circuits (e.g., the stomatogastric circuit of the lobster) and have been shown to contribute to the production of rhythmic output (Russell and Hartline, 1978; Raper, 1979; Selverston and Miller, 1980; Miller and Selverston, 1982b). Another property necessary for the oscillation is a time-varying restorative property that removes the inhibitory effect of one neuron onto the other and permits the transition from inactive 

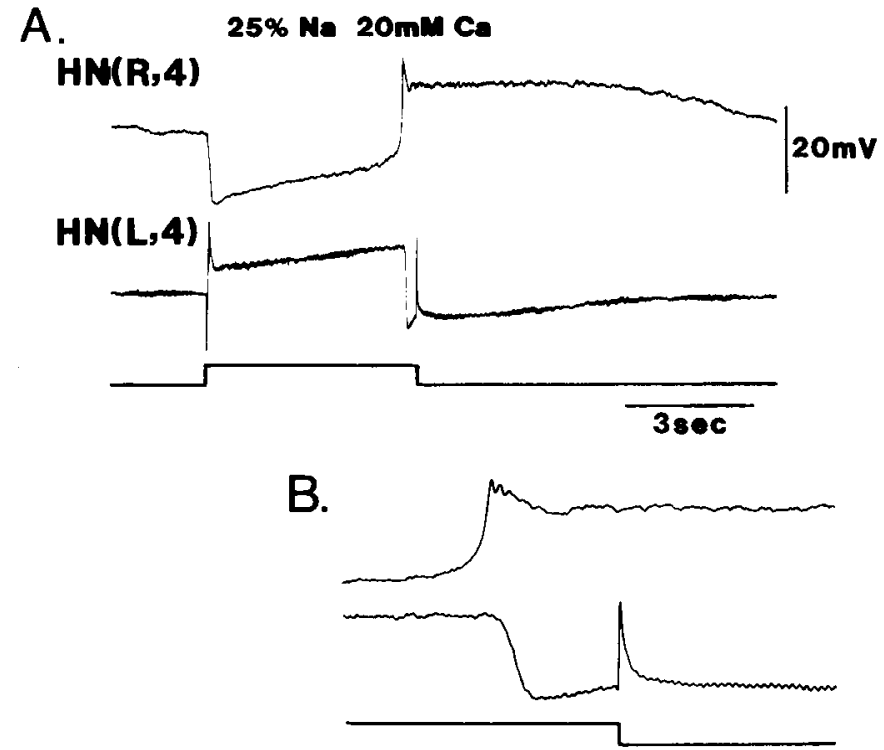

Figure 6. Escape of an $\mathrm{HN}$ interneuron from inhibition. The preparation was bathed in saline containing $25 \% \mathrm{Na}^{+}$and $20 \mathrm{~mm} /$ liter $\mathrm{Ca}^{2+}$. $A$, Depolarization of the $\mathrm{HN}(\mathrm{L}, 4)$ by an injected current pulse caused synaptic inhibition of the HN $(\mathrm{R}, 4)$. The membrane potential of the inhibited HN neuron moved to an initial maximum level of hyperpolarization, then gradually relaxed to more depolarized levels. It ultimately escaped the synaptic inhibition and formed a plateau, despite continuing stimulus to the contralateral $\mathrm{HN}$ interneuron. $B$, The transition to a plateau is shown more clearly in the expanded trace. Plateau formation in HN $(R, 4)$ led to synaptic inhibition of the HN $(L, 4)$ prior to the termination of the depolarizing current marked by the current monitor in the lower trace and the capacitive "off" transient in the recording of $\mathrm{HN}(\mathrm{L}, 4)$. The injected current was $0.25 \mathrm{nA}$.

to active (hyperpolarized to depolarized) states. Processes thought to mediate this function in different systems include synaptic fatigue, such as has been demonstrated in stomatogastric neurons (Hartline and Gassie, 1979), and spike frequency adaptation, such as has been found in neurons of the Tritonia swim system (Hume and Getting, 1982) and postinhibitory rebound (Perkel and Mulloney, 1974; Satterlie, 1985). In the heartheat timing oscillator, we have shown that escape from inhibition times the phase transitions of strongly oscillating $\mathrm{HN}$ interneurons and that a hyperpolarization-activated restorative shift in membrane potential may contribute significantly to the escape of one $\mathrm{HN}$ interneuron from inhibition by the other.

Although these mechanisms can be shown to be responsible for the plateau-based oscillation under altered ionic conditions, an important question is how they relate to oscillation of this system in normal saline in the presence of action potentials. Examination of normal cycling of HN neurons of the timing oscillator (Fig. $1 B$ ) indicates that, while there is a slow wave of polarization accompanying each cycle of activity and inhibition, the depolarizing phase of the cycle does not include a plateau potential of the type exhibited in the absence of $\mathrm{Na}^{+}$. It is likely, however, that conductances responsible for the formation of plateau potentials in the absence of $\mathrm{Na}^{+}$are activated in the depolarized phase of the cycle. Formation of a plateau may be prevented by shunting due to IPSPs caused by firing of the contralateral HN neuron or due to the afterhyperpolarizations of action potentials produced during the burst. Depending on the magnitude of the hyperpolarization produced by inhibitory input from the contralateral neuron and the rate of depolarization during escape from inhibition, plateau-forming conductance may be activated to different degrees under different conditions of oscillation. Termination of a large injected hyperpolarizing pulse activates a plateau in spite of the ongoing production of action potentials (Arbas and Calabrese, 1987; Fig. $1 B$ ). Any amount of the plateau-forming conductance activated under normal conditions would depolarize the neuron and support the generation of $\mathrm{Na}^{+}$action potentials.

The hyperpolarized phase of the activity cycle in each $\mathrm{HN}$ neuron is governed by multiple parameters. The magnitude of the hyperpolarization depends on the frequency of firing during the burst of the contralateral neuron and the magnitude of the slow wave, as well as other factors such as presynaptic modulation of neurotransmitter release (Thompson and Stent, 1976c; Nicholls and Wallace, 1978). We have also shown that a restorative shift in membrane potential is likely to be activated during this phase of the activity cycle through the hyperpolarization due to synaptic inhibition. Since the restorative shift is hyperpolarization sensitive, it could also be activated by any increases in $\mathrm{K}^{+}$currents.

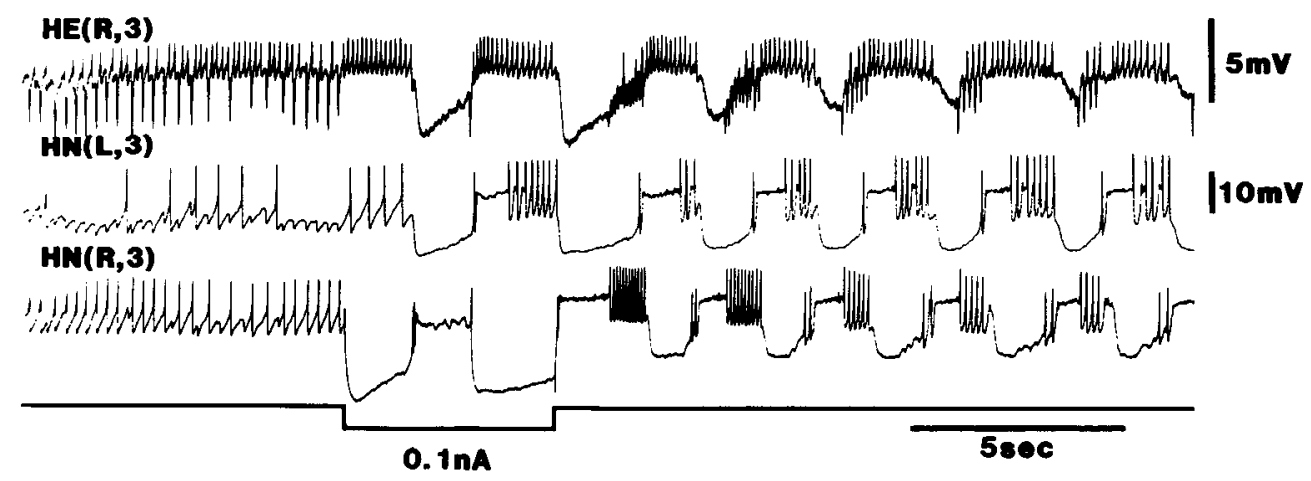

Figure 7. Restorative current is sufficient to initiate oscillation. Simultaneous recordings from both timing oscillator interneurons of ganglion 3 and a motorneuron postsynaptic to one of them. Following reduction of $\mathrm{Na}^{+}$by half, but in the presence of normal Ca ${ }^{2+}$, the oscillation ceased (beginning of traces). A hyperpolarizing current pulse injected into one HN neuron activated the restorative sag in membrane potential. When the membrane potential reached a sufficiently depolarized level, a plateau was formed despite continuing injection of hyperpolarizing current. Inhibition of both the contralateral interneuron and the motorneuron followed. The contralateral interneuron escaped from inhibition, formed a plateau of its own and inhibited the plateau of the stimulated neuron, completing a full cycle of oscillation. Release of the $H N(R, 3)$ from the injected current triggered another plateau and set off oscillation of the network. 

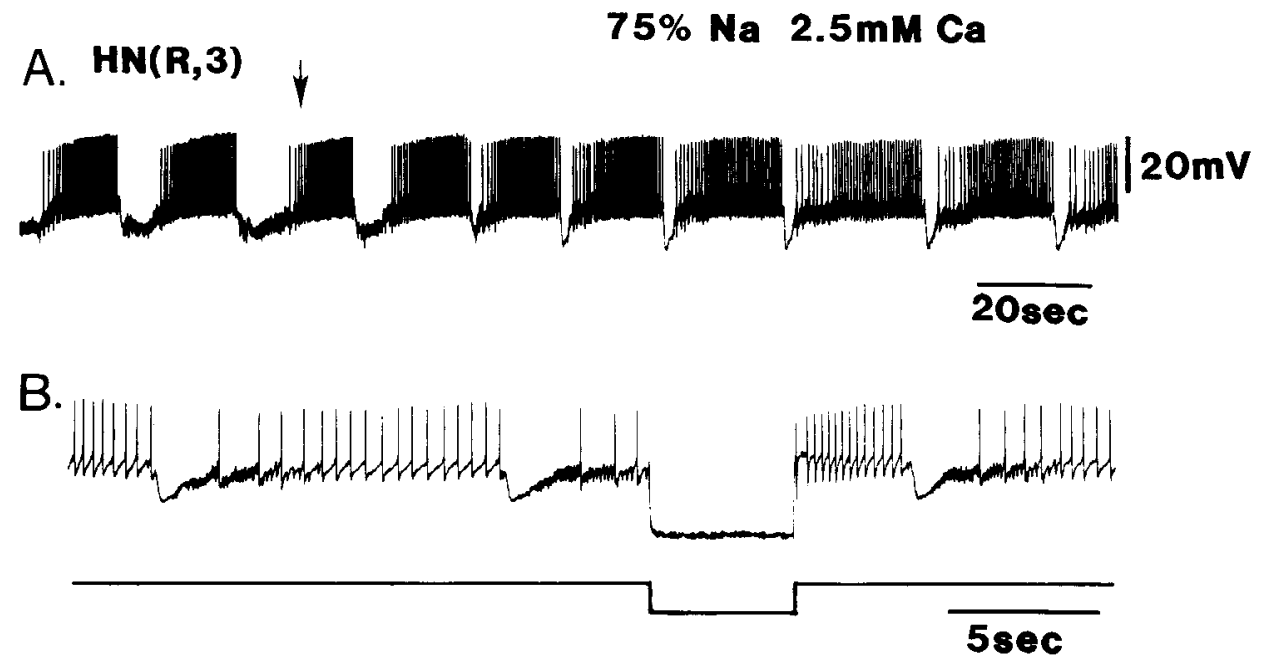

Figure 8. $\mathrm{Cs}^{+}$slows, but does not halt, the oscillation of the timing oscillator. $A$, Recording from one of an oscillating pair of HN (3) interneurons shows the effect of adding $\mathrm{Cs}^{+}$-containing saline (arrow). Slowing of cycle rate and prolongation of burst duration were evident within $20 \mathrm{sec}$ of adding $\mathrm{Cs}^{+}$to the saline, though oscillation continued for the duration of the recording (several minutes). $B$, Recording from the same HN neuron 4 min after the end of the trace shown in $A$. Injection of a hyperpolarizing current pulse showed no trace of a restorative sag in membrane potential. Pulse, $0.1 \mathrm{nA}$. The ganglion was bathed in saline containing $75 \% \mathrm{Na}^{+}$ and $2.5 \mathrm{~mm} /$ liter $\mathrm{Ca}^{2+}$, to which the 5 $\mathrm{mm} /$ liter $\mathrm{Cs}^{+}$was added.
The timing of phase transitions in strongly oscillating pairs of HN cells is controlled by escape from inhibition of the hyperpolarized neuron. Termination of bursts of activity in HN cells (3) or (4) in normal saline are preceded by onset of activity in their contralateral homologs (Peterson 1983a, b). It was suggested by Peterson (1983) that an endogenous property of $\mathrm{HN}$ cells that makes them refractory to inhibition permits this recovery. We suggest here that the conductances mediating hyperpolarization-activated shifts in membrane potential contribute significantly to escape from inhibition.

$\mathrm{HN}$ interneurons have been shown to have the capability for endogenous bursting (Calabrese, 1979), yet plateau-based oscillations could be silenced and spontaneous oscillation seemed to be absent under certain experimental conditions of this study (Fig. 8). All of the components necessary for $\mathrm{HN}$ neurons to oscillate endogenously were evident in these experiments, except an endogenous pacemaker potential. If the pacemaker potential were dependent on $\mathrm{Na}^{+}$, it could have been eliminated or greatly weakened in its ability to drive plateauing under the experimental conditions reported here. In reduced $\mathrm{Na}^{+}$salines, the driving force for the current would be eliminated. In solutions containing elevated concentrations of divalent ions, the voltage dependence of $\mathrm{Na}^{+}$conductances was altered such that a pacemaker current might be rendered inoperative over the voltage ranges of plateau-based oscillation. It has been shown clearly in other systems that some neurons depend on tonic exogenous inputs for the expression of their rhythmical properties (Miller and Selverston, 1982a; Dickinson and Nagy, 1983; Nagy and Dickinson, 1983; Moulins and Nagy, 1985). Removal of $\mathrm{Na}^{+}$ ions from the saline or elevating divalent ions, as done in our experiments, reduced or eliminated the spontaneous firing of action potentials in all other neurons of the ganglion as well as of the HN cells. This could have had the effect of blocking exogenous inputs necessary for expression of rhythmic properties.

\section{Exogenous inputs}

We have shown in other studies that the rate of the heartbeat rhythm can vary over nearly an order of magnitude under different conditions (Arbas and Calabrese, 1984). Additionally, the timing oscillator is known to be under modulatory control by several identified neural groups outside of the heartbeat pattern generator (Arbas and Calabrese, 1983, 1984; Kuhlman et al.,
1985). Thus, the relative importance of some of the parameters that cooperate to produce oscillation may vary at different rates of cycling, and some parameters are likely to come under exogenous control in mediating the observed changes in rate. Exogenous adrenergic inputs have been shown to govern cardiac pacemaking, at least in part, through their action on the kinetics of the hyperpolarization-activated inward current, $I_{\mathrm{f}}$ (DiFrancesco, 1985, 1987). Increases in the depolarizing tendency of the restorative sag in membrane potential initiated by hyperpolarization of $\mathrm{HN}$ interneurons may, similarly, translate into an acceleration in the rate of oscillation of the timing oscillator and, therefore, the entire heartbeat network.

\section{References}

Arbas, E. A., and R. L. Calabrese (1983) Leydig cell activity modulates heartbeat pattern generator cycling in the leech. Soc. Neurosci. Abstr. 9: 755.

Arbas, E. A., and R. L. Calabrese (1984) Rate modification in the heartbeat central pattern generator of the medicinal leech. J. Comp. Physiol. 155: 783-794.

Arbas, E. A., and R. L. Calabrese (1987) Ionic conductances underlying the activity of interneurons that control heartbeat in the medicinal leech. J. Neurosci. 7: 3945-3952.

Brown, T. A. (1914) On the nature of the fundamental activity of the nervous centres together with an analysis of the conditioning of the rhythmic activity in progression and a theory of the evolution of function in the nervous system. J. Physiol. (Lond.) 48: 18-46.

Calabrese, R. L. (1979) The roles of endogenous membrane properties and synaptic interaction in generating the heartbeat rhythm of the leech. Hirudo medicinalis. J. Exp. Biol. 82: 163-176.

Calabrese, R. L., and E. L. Peterson (1983) Neural control of heartbeat in the leech, Hirudo medicinalis. In Neural Origin of Rhythmic Movements. A. Roberts and B. Roberts, eds. Symp. Soc. Exp. Biol. 37: 195-221.

Delcomyn, F. (1980) Neural basis of rhythmic behavior in animals. Science 210: 492-498.

Dickinson, P. S., and F. Nagy (1983) Control of a central pattern generator by an identified modulatory interneurone in crustacea. II. Induction and modification of plateau properties in pyloric neurones. J. Exp. Biol. 105: 59-82.

DiFrancesco, D. (1985) The cardiac hyperpolarizing-activated current, $\mathrm{I}_{\mathrm{f}}$. Origins and developments. Prog. Biophys. Mol. Biol. 46: 163-183.

DiFrancesco, D. (1987) Characterization of single pacemaker channels in cardiac sino-atrial node cells. Nature $324: 470-473$.

Frankenhaeuser, B., and A. L. Hodgkin (1957) The action of calcium on the electrical properties of squid axons. J. Physiol. (Lond.) 137: 218-244.

Hartline, D. K., and D. V. Gassie (1979) Pattern generation in the 
lobster panilurus stomatogastric ganglion. I. Pyloric neuron kinetics and synaptic interactions. Biol. Cybernet. 33: 209-222.

Hume, R. I., and P. A. Getting (1982) Motor organization of Tritonia swimming. III. Contribution of intrinsic membrane properties to flexion neuron burst formation. J. Neurophys. 47: 91-102.

Kuhlman, J. R., C. Li, and R. L. Calabrese (1985) FMRF-amide-like substances in the leech II. Bioactivity on the heartbeat system. J. Neurosci. 5: 2310-2317.

Miller, J. P., and A. I. Selverston (1982a) Mechanisms underlying pattern generation in lobster stomatogastric ganglion as determined by selective inactivation of identified neurons. II. Oscillatory properties of pyloric neurons. J. Neurophysiol. 48: 1378-1391.

Miller, J. P., and A. I. Selverston (1982b) Mechanisms underlying pattern generation in lobster stomatogastric ganglion as determined by selective inactivation of identified neurons. IV. Network properties of pyloric system. J. Neurophysiol. 48: 1416-1432.

Moulins, M., and F. Nagy (1985) Extrinsic inputs and flexibility in the motor output of the lobster pyloric neural network. In Model Neural Networks and Behavior, A. I. Selverston, ed., pp. 49-68, Plenum, New York.

Nagy, F., and P. S. Dickinson (1983) Control of a central pattern generator by an identified modulatory interneurone in crustacea. 1 . Modulation of the pyloric motor output. J. Exp. Biol. 105: 33-58.

Nicholls, J. G., and B. G. Wallace (1978) Modulation of transmission at an inhibitory synapse in the central nervous system of the leech. J. Physiol. (Lond.) 281: 157-170.

Perkel, D. H., and B. Mulloney (1974) Motor pattern production in reciprocally inhibitory neurons postinhibitory rebound. Science 185 : 181-183.

Peterson, E. L. (1983a) Generation and coordination of heartbeat timing oscillation in the medicinal leech. I. Oscillation in isolated ganglia. J. Neurophysiol. 49: 611-626.
Peterson, E. L. (1983b) Generation and coordination of heartbeat timing oscillation in the medicinal leech. II. Intersegmental coordination. J. Neurophysiol. 49: 627-638.

Peterson, E. L., and R. L. Calabrese (1982) Dynamic analysis of a rhythmic neural circuit in the leech, Hirudo medicinalis. J. Neurophysiol. 47: 256-271.

Raper, J. (1979) Non-impulse mediated synaptic transmission during the generation of a cyclic motor program. Science 205: 304-306.

Roberts, A., and B. Roberts (1983) Neural Origin of Rhythmic Movements. Symp. Soc. Exp. Biol. 37.

Russell, D. F., and D. K. Hartline (1978) Bursting neural networks: A reexamination. Science 200: 453-456.

Satterlie, R. A. (1985) Reciprocal inhibition and postinhibitory rebound produce reverberation in a locomotor pattern generator. Science 229: 402-404.

Selverston, A. I., and J. P. Miller (1980) Mechanisms underlying pattern generation in lobster stomatogastric ganglion as determined by selective inactivation of identified neurons. I. Pyloric system. J. Neurophysiol. 44: 1102-1121.

Selverston, A. I., and M. Moulins (1985) Oscillatory neural networks. Annu. Rev. Physiol. 47: 29-48.

Thompson, W. J., and G. S. Stent (1976a) Neuronal control of heartbeat in the medicinal leech. I. Generation of the vascular constriction rhythm by heart motor neurons. J. Comp. Physiol. 111: 261-279.

Thompson, W. J., and G. S. Stent (1976b) Neuronal control of heartbeat in the medicinal leech. II. Intersegmental coordination of heart motor neuron activity by heart interneurons. J. Comp. Physiol. 111: 281-307.

Thompson, W. J., and G. S. Stent (1976c) Neuronal control of heartbeat in the medicinal leech. III. Synaptic relations of the heart interneurons. J. Comp. Physiol. 111: 309-333. 